\title{
Performance Analysis of a Full-Duplex Energy Harvesting Cognitive Wireless Network
}

\author{
Hebert D. Pereira, Samuel B. Mafra, and Evelio M. G. Fernandez
}

\begin{abstract}
The performance of a full-duplex cooperative cognitive network is investigated under Nakagami- $m$ fading. The secondary source and the relays are energy-constrained nodes and harvest energy from the primary network to transmit their information. The direct link between source and destination in the secondary network is considered as useful information at destination where joint-decoding is applied upon the signals received from source and relay. We derive an approximated closed-form expression for the outage probability of the proposed scheme. Results show that the proposed scheme outperforms the half-duplex and direct transmission schemes in the particular scenarios under consideration. Moreover, the performance in terms of outage probability of the proposed scheme is further improved through the use of multiple relays.
\end{abstract}

Index Terms-Energy Harvesting, cooperative cognitive network, full-duplex communication, joint-decoding.

\section{INTRODUCTION}

O VER the last few decades, the continuous technological advances have enabled the exponential growth of wireless networks and several techniques have been proposed to use radio frequency (RF) spectrum and energy more efficiently as both are limited natural resources. Among these techniques, Cognitive Radio (CR) is a promising technology that provides a basis for addressing the practical issue of spectrum scarcity [2]. In a cognitive radio network, unlicensed or secondary users (SUs) can use the same frequency band allocated to licensed or primary users (PUs) as long as the interference caused to the PUs is below a predefined threshold [3].

In addition, in order to improve the energy efficiency in wireless networks, the concept of energy harvesting $(\mathrm{EH})$ is becoming a promising solution, considering the high maintenance cost of these networks. [4]. EH is the process of extracting energy from the surrounding environment in order to power and to prolong the lifetime of energy-constrained communication networks. A variety of energy sources such as heat, light, wave, and wind have been considered for EH in wireless networks [4]-[6], but this sources depends on weather conditions. More recently, energy harvested from RF signals has received an increasing attention due to its convenience in providing energy self-sufficiency and independence of weather conditions [7].

Hebert D. Pereira, Samuel B. Mafra and Evelio M. G. Fernandez are with the Graduate Program in Electrical Engineering, Federal University of Paraná (UFPR), Curitiba, Brazil, (e-mail: hebert.douglas@gmail.com, mafrasamuel@gmail.com, evelio@ufpr.br)

This work has been partially supported by CAPES and Araucaria Foundation.

A preliminary version of this paper was presented in XXXV Simpósio Brasileiro de Telecomunicações (SBrT'17), São Pedro, SP, Brazil, September 3-6, 2017 [1]

Digital Object Identifier: 10.14209/jcis.2018.23
Two commonly used EH protocols are time switching (TS) and power splitting (PS) [8]-[10]. In the TS protocol, a fraction of time is used to harvest energy from the received signal and the rest of the time is used to receive/send information from/to the destination. While in the PS protocol, a fraction of the received signal power is used for harvesting energy and the remainder is used to receive/send information from/to the destination [8].

On the other hand, in scenarios with size, cost, or hardware limitations, such as wireless sensor networks, cooperative communication has emerged as a key enabling technology for efficient spectrum usage [11], [12]. In a cooperative communication link, a relay node cooperates with the communication from source to destination, so the destination receives two different versions of the transmitted signal that can be combined, allowing the system to achieve transmit diversity.

The relay can operate in half-duplex (HD) mode, in which transmission and reception occur at different time-slots or in full-duplex (FD) mode, in which transmission and reception are performed at the same time and at the same frequency band. Consequently, FD relaying can achieve higher capacity than HD relaying [13], however in FD mode the communication is affected by self-interference since perfect isolation between transmit and receive antennas at the relay can not be guaranteed [14], [15]. Additionally, there are mainly two relaying protocols: amplify-and-forward (AF) and decodeand-forward (DF). In AF, the relay simply amplifies and retransmits the information to the destination, so the noise is also amplified. In DF, the relay first attempts to decode the received signal and then, if successful, the information is re-encoded and retransmitted to the destination.

The use of wireless energy harvesting using RF signal has also been studied in the context of cooperative communications. In [10], the time switching relaying (TSR) and the power splitting relaying (PSR) protocols were proposed for an AF relaying network in which an energy-constrained relay harvests energy from the received RF signal and uses this energy to forward the information received from the source to the destination. In [16], performance measures such as average signal-to-noise ratio (SNR), outage probability and throughput are analyzed in a wireless energy harvesting relay network with multiple relays. A relay selection scheme is also proposed to achieve the full diversity at any EH time.

In [17] a dual-hop FD relaying network is analyzed where the energy constrained relay node is powered by RF signals from the source using the TS harvesting protocol and considering both the AF and DF relaying protocols. The results show that, when the time for energy harvesting is optimized, the FD relaying could substantially boost the system throughput 
compared to the conventional HD relaying architecture.

Cooperative cognitive radio networks have also been widely addressed in the literature. For instance in [18], the authors examine the impact of multiple primary transmitter and receivers on the performance, in terms of outage probability, of cognitive DF-HD relay networks. In [19], a new FD relaying scheme is proposed for a cognitive network where the secondary destination jointly decodes the signals from the secondary transmitter and from the FD secondary relay. Furthermore, the authors consider the signal received form the direct link as useful information, rather than interference. Results show that the proposed FD scheme presents the best performance among all schemes investigated in the paper. In [20], the scenario presented in [18] is extended to an EH scenario in which the secondary nodes harvest energy from multiple primary transmitters. The results show that when the number of primary transmitters is large, the detrimental effect of the interference from the PUs outweighs the benefits of the energy that can be harvested from them.

In this paper we extend the scenario presented in [19] to a scenario in which both the source and relay nodes are energyconstrained and harvest energy from the RF signals transmitted by the PU to support information transmission as in [20]. Therefore, a FD relaying cognitive radio network is considered. To the best of the authors knowledge, such a scenario has not yet been proposed in the literature. The contribution of this paper is twofold: first, an approximated expression is derived for the outage probability of the secondary network in a FD relaying cognitive radio network; and secondly, the performance in terms of outage probability of the proposed scheme is further improved through the use of multiple relays.

The rest of the paper is organized as follows. In Section II, the system model and assumptions regarding the EH protocol are described. In Section III, approximated expressions for the outage probability are given while in Section IV, numeric results and discussions are presented. Section V concludes the paper.

\section{SySTEM MOdEL}

A cognitive cooperative network is considered in which an energy-constrained source $\mathrm{S}$ transmits to a self-powered destination $\mathrm{D}$ with the help of multiple energy-constrained relays $\mathrm{R}_{u}, u \in\{1,2, \cdots, U\}$. The primary network consists of one primary transmitter $\mathrm{P}^{1}$ which is near the secondary network so, an interference-limited scenario is taken under consideration as in [18]. Fig. 1 illustrates the proposed scenario in which only the selected relay $\mathrm{R}_{*}$ is shown.

Differently from [16]-[18], [20], we consider the signal received from the direct link between $\mathrm{S}$ and $\mathrm{D}$ as useful information. Consequently, joint-decoding is performed at $\mathrm{D}$ upon the signals received from $S$ and $R_{*}$ as in [19]. Both $S$ and $\mathrm{R}_{*}$ transmit their information using only the energy harvested from the primary RF signal ${ }^{2}$ using the TS-EH protocol. All

\footnotetext{
${ }^{1}$ It is assumed that the SUs are far enough from the primary destination so that the interference caused by the SUs to the primary destination is negligible.

${ }^{2}$ It is assumed that the nodes are provided with a battery capable of keeping their circuitry active, otherwise the problem becomes very difficult to be modeled as showed in [21].
}

the energy harvested by the nodes is stored in a rechargeable battery, that can be a super-capacitor or a high-efficiency battery and then used for information transmission in just one time slot as in [10], [21].

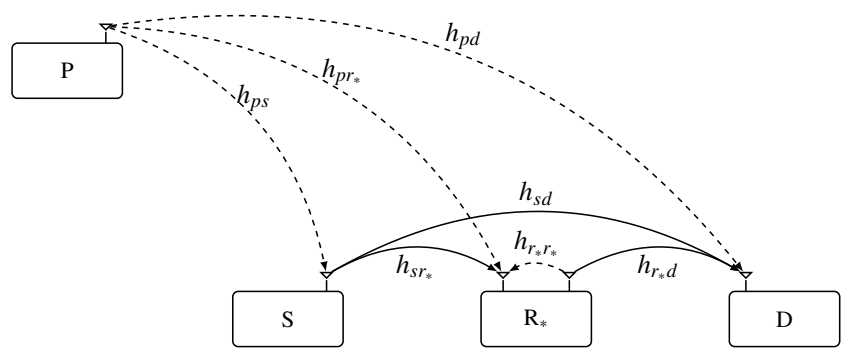

Fig. 1: Cognitive Cooperative Full-Duplex Network with Multiple Relays

The quasi-static fading channel between transmitter $i$ and receiver $j$ is denoted by $h_{i j}, i \in\left\{p, s, r_{*}\right\}, j \in\left\{s, r_{*}, d\right\}$, whose envelope follows a Nakagami- $m$ distribution ${ }^{3}$ with shape parameter $m_{i j}$. The average channel power gain is $\lambda_{i j} \triangleq \mathbb{E}\left[\left|h_{i j}\right|^{2}\right] \triangleq \hat{d}_{i j}^{-v}$, where $\hat{d}_{i j}=\frac{d_{i j}}{d_{p d}}$ is the normalized distance between the primary transmitter and the secondary destination, and $v$ is the path-loss exponent $(v \geq 2)$. The residual self-interference at the FD relay is modeled by a circularly symmetric complex Gaussian distribution with zero mean and variance $\sigma_{r_{*} r_{*}}^{2}$. Consequently, the channel between the two antennas of the the FD relay can be seen as a fading channel with coefficient $h_{r_{*} r_{*}} \sim \mathcal{C N}\left(0, \sigma_{r_{*} r_{*}}^{2}\right)$ and average power $\lambda_{r_{*} r_{*}} \triangleq \delta$, where $\delta$ represents the interference cancellation factor which arises from the association of antenna isolation and interference cancellation techniques [14].

\begin{tabular}{c|c|c|}
\hline$\underbrace{\mathrm{P} \rightarrow\left(\mathrm{S}, \mathrm{R}_{1}, \cdots, \mathrm{R}_{U}\right)}_{\text {Time }} \mid \mathrm{S} \rightarrow\left(\mathrm{R}_{1}, \cdots, \mathrm{R}_{U}, \mathrm{D}\right)$ & $\mathrm{R}_{*} \rightarrow \mathrm{D}$ \\
\hline$\alpha T$ Harvesting &
\end{tabular}

Fig. 2: HD protocol for energy harvesting and information transmission.

$\underbrace{\underbrace{\mathrm{P} \rightarrow\left(\mathrm{S}, \mathrm{R}_{1}, \cdots, \mathrm{R}_{U}\right)}_{\alpha T \text { Harvesting }}}_{\text {Time }} \underbrace{\mathrm{S} \rightarrow\left(\mathrm{R}_{1}, \cdots, \mathrm{R}_{U}, \mathrm{D}\right) \text { and } \mathrm{R}_{*} \rightarrow \mathrm{D}}_{(1-\alpha) \beta T \text { Information }}$

Fig. 3: FD protocol for energy harvesting and information transmission.

In this paper, we consider the TS energy harvesting protocol, where $\mathrm{S}$ and relays first harvest energy from $\mathrm{P}$ during a fraction $\alpha T$ of each time-slot of duration $T$, as shown in Figures 2 and

\footnotetext{
${ }^{3}$ Nakagami- $m$ is a general distribution that fits several types of fading. The Rayleigh distribution, for example, corresponds to $m=1$. For $m>1$ there is some line-of-sight between transmitter and receiver.
} 
3. After that, $\mathrm{S}$ and $\mathrm{R}_{*}$ transmit their information during a time $(1-\alpha) \beta T$, being $\beta=\frac{1}{2}$ for the HD mode (Fig. 2), while $\beta=1$ for the FD mode (Fig. 3) and for the direct transmission which will be also considered for comparison purposes. Therefore, the energy harvested at node $k\left(k \in\left\{s, r_{*}\right\}\right)$ is given by

$$
E_{k}=\eta P_{p}\left|h_{p k}\right|^{2} \alpha T
$$

where $\eta(0 \leq \eta \leq 1)$ is the energy transfer efficiency and $P_{p}$ is the primary transmit power. Consequently, the available transmit power at node $k$ is

$$
P_{k}=\frac{E_{k}}{\beta(1-\alpha) T}=\frac{\alpha \eta P_{p}}{\beta(1-\alpha)}\left|h_{p k}\right|^{2}=\mathcal{K} P_{p}\left|h_{p k}\right|^{2},
$$

where $\mathcal{K}=\frac{\alpha \eta}{\beta(1-\alpha)}$.

The signals received at $\mathrm{R}_{*}$ and $\mathrm{D}$ at time $t$ can be expressed, respectively, as

$$
\begin{aligned}
& y_{r_{*}}[t]=\sqrt{P_{s}} h_{s r_{*}} x_{s}[t]+\sqrt{P_{r_{*}}} h_{r_{*} r_{*}} \hat{x}_{r}[t]+\sqrt{P_{p}} h_{p r_{*}} x_{p}[t], \\
& y_{d}[t]=\sqrt{P_{r_{*}}} h_{r_{*} d} \hat{x}_{r}[t]+\sqrt{P_{s}} h_{s d} x_{s}[t]+\sqrt{P_{p}} h_{p d} x_{p}[t],
\end{aligned}
$$

where $P_{i}$ is the transmit power at node $i, x_{s}[t]$ is the message sent by $\mathrm{S}, x_{p}[t]$ is the message sent by $\mathrm{P}$, and $\hat{x}_{r}[t] \triangleq x_{s}[t-d]$ is the message retransmitted by $\mathrm{R}_{*}$ after a processing delay $d$.

\section{Outage Probability AnAlysis}

We consider as performance metric the outage probability. Outage is the event that the mutual information between nodes $i$ and $j$ is less than an attempted information rate $\mathcal{R}_{\text {sch }}$. Assuming unit bandwidth, the outage probability of the link $k \rightarrow j$, with $k \neq j$, is obtained as

$$
O_{k j}=\operatorname{Pr}\left\{\log _{2}\left(1+\frac{\mathcal{K}\left|h_{k j}\right|^{2}\left|h_{p k}\right|^{2}}{\left|h_{p d}\right|^{2}}\right)<\frac{\mathcal{R}_{\mathrm{sch}}}{1-\alpha}\right\},
$$

where $\operatorname{Pr}\{a\}$ is the probability of event $a$ and $\mathcal{R}_{\text {sch }}=\frac{\mathcal{R}}{\mathrm{R}_{\mathrm{sch}}}$ is the transmission rate in bits per channel use (bpcu) of the scheme sch $\in\{$ EH-DT, EH-HDJD, EH-FDJD $\}$ and $\mathcal{R}$ is the attempted information rate in the case of non-cooperative direct transmission. Note that $\mathrm{R}_{\mathrm{sch}}$ corresponds to the code rate (the ratio between the number of information packets and the total number of packets) of a given scheme sch, where $\mathrm{R}_{\mathrm{EH}-\mathrm{FDJD}}=\mathrm{R}_{\mathrm{EH}-\mathrm{DT}}=1$ for FD and direct transmissions and $\mathrm{R}_{\mathrm{EH}-\mathrm{HDJD}}=\frac{1}{2}$ for HD transmission.

\section{A. Energy Harvesting Direct Transmission Scheme}

In the Energy Harvesting Direct Transmission Scheme (EHDT), the source devotes a fraction $\alpha$ of time slot to harvest energy from the primary transmission and transmit a new information in the remainder of the time-slot.

The outage probability of EH-DT scheme can be expressed as

$$
\begin{aligned}
O_{s d}^{\mathrm{EH}-\mathrm{DT}} & =\operatorname{Pr}\left[\mathcal{I}_{s d}<\mathcal{R}_{\mathrm{sch}}\right] \\
& =\operatorname{Pr}\left[(1-\alpha) \log _{2}\left(1+\frac{P_{s}\left|h_{s d}\right|^{2}}{P_{p}\left|h_{p d}\right|^{2}}\right)<\mathcal{R}_{\mathrm{EH}-\mathrm{DT}}\right] \\
& =\operatorname{Pr}\left[\log _{2}\left(1+\frac{\mathcal{K}\left|h_{p s}\right|^{2}\left|h_{s d}\right|^{2}}{\left|h_{p d}\right|^{2}}\right)<\frac{\mathcal{R}_{\mathrm{EH}-\mathrm{DT}}}{(1-\alpha)}\right] .
\end{aligned}
$$

However, the outage probability of the EH-DT scheme presented in (6) has not a general closed-form expression. In order to evaluate the effects of energy harvesting on the performance of the proposed scheme, we derive approximated closed-form expressions for two scenarios of interest with some particular fading parameters.

1) In the first scenario, we are interested in evaluate the effect of some line-of-sight in the harvesting and interference links. We consider $m_{k j}=1$ (Rayleigh fading) for the link $k \rightarrow j$ and generic values of fading parameter for the links between the primary network and the secondary network $\left(m_{p k} \geq 1\right.$ and $\left.m_{p j} \geq 1\right)$;

2) In the second scenario, we evaluate the effect of some line-of-sight in a link of the secondary network. We consider $m_{p k}=m_{p j}=1$ (Rayleigh fading) for the link between the primary and secondary networks, and a generic value for the fading parameter $m_{k j} \geq 1$ for the link $k \rightarrow j$ with $k \neq j$;

Following the analysis in [22], we can write the outage probability of the link $S \rightarrow D$, taking into account the two scenarios above as in (7), where $\mathrm{E}_{n}(n, z)=\int_{1}^{\infty} \frac{e^{-z t}}{t^{n}} d t$ is the exponential integral [23, Eq. (5.1.4)], $U(a, b, c)$ is the confluent hypergeometric function [24, Eq. (9.211.4)] and $\mathcal{Z}_{m d}$ is a constant given by:

$$
\mathcal{Z}_{m d}=\frac{\left(2^{\frac{\mathcal{R}_{\mathrm{sch}}}{(1-\alpha)}}-1\right) m_{s d} \lambda_{p d}}{\mathcal{K} \lambda_{p s} \lambda_{s d}}
$$

\section{B. Relay Selection Strategy}

We assume perfect knowledge of the channels in all nodes of the secondary network that can be obtained through pilot signals according to [25]-[27].

A distributed relay selection protocol is adopted based on a reactive mode, where the selected relay is the one that maximizes the instantaneous channel gain between the relay and the destination, among all the relay that correctly decoded the source message. Thus, the relay that decoded the previous source information and have a better channel gain between $R$ and $\mathrm{D}$ will be the one chosen to transmit to the destination, as proposed in [28], [29]. So the selected relay is expressed as

$$
R_{*}=\underset{u \in\{1, \ldots, U\}}{\arg \max }\left(\left|h_{r_{u} d}\right|^{2}\right) .
$$

In a distributed relay selection, each relay waits a time proportional to a control variable that is given as a function of the gain of the channel before perform its transmission. The control variable is given by $t_{c} \sim \frac{1}{\left|h_{r_{u} d}\right|^{2}}+\varphi$. As the gain of the channels can present identical values for different relays, we include a Gaussian random variable $\varphi$ to avoid simultaneous transmissions of more than one relay. The variable $\varphi$ has zero mean and variance $\sigma_{\varphi}^{2}$, which value is very small compared to channel gain $\left|h_{r_{u} d}\right|^{2}$. Moreover, we assume that the other relays overhear the transmission and remain silent to avoid collisions. 


$$
\begin{aligned}
& O_{s d}^{\mathrm{EH}-\mathrm{DT}}=\int_{0}^{\infty} \int_{0}^{\infty} \int_{0}^{\frac{z_{p s}\left(2 \frac{\mathcal{R}_{\mathrm{EH}-\mathrm{DT}}}{(1-\alpha)}-1\right)}{K_{z p s}}} \frac{e^{-\frac{m_{s d} z_{s d}}{\lambda_{s d}}\left(\frac{m_{s d} z_{s d}}{\lambda_{s d}}\right)^{m_{s d}}}}{z_{s d} \Gamma\left(m_{s d}\right)} \frac{e^{-\frac{m_{p d} z_{p d}}{\lambda_{p d}}}\left(\frac{m_{p d} z_{p d}}{\lambda_{p d}}\right)^{m_{p d}}}{e^{-\frac{m_{p s} z_{p s}}{\lambda_{p s}}}\left(\frac{m_{p s} z_{p s}}{\lambda_{p s}}\right)^{m_{p s}}} \frac{\left.\mathrm{d} m_{p d}\right)}{z_{p s} \Gamma\left(m_{p s}\right)} \mathrm{d} z_{p d} \mathrm{~d} z_{p s} \\
& = \begin{cases}\mathcal{Z}_{m d} e^{\mathcal{Z}_{m d}} \mathrm{E}_{n}\left(m_{s d}, \mathcal{Z}_{m d}\right), & \text { for } m_{s d}=1 \text { and } m_{p s}=m_{p d}=1 \\
1-\frac{\Gamma\left(m_{p s}+m_{p d}\right) U\left(m_{p d}, 1-m_{p s}, \frac{Z_{m d} m_{p s}}{m_{p d}}\right)}{\Gamma\left(m_{p s}\right)}, & \text { for } m_{s d}=1, m_{p s} \geq 1 \text { and } m_{p d} \geq 1 .\end{cases}
\end{aligned}
$$

\section{Energy Harvesting Full-Duplex with Joint-Decoding Scheme}

In the proposed Energy Harvesting Full-duplex with JointDecoding scheme (EH-FDJD), whose protocol is showed in Fig. 3, the broadcast and cooperation phases occur at the same time. The source broadcast its information to the relays and to destination, while the selected relay forwards a previous information of source to the destination after a processing delay. The communication through the direct link is also considered as useful information, so when the source-relay link is in outage, the message can still be successfully received through the direct link. Finally, at the destination both messages are combined using the joint decoding scheme [19], [30], which is an iterative process for decoding information blocks based on the knowledge acquired from previously received blocks.

As a result, the mutual information of the links $S \rightarrow R_{*}$ and $\mathrm{R}_{*} \rightarrow \mathrm{D}$ can be computed, respectively, as:

$$
\begin{aligned}
& \mathcal{I}_{s r_{*}}^{F D}=\mathrm{R}_{\mathrm{EH}-\mathrm{FDJD}}(1-\alpha) \log _{2}\left(1+\frac{P_{s}\left|h_{s r_{*}}\right|^{2}}{P_{p}\left|h_{p r_{*}}\right|^{2}+P_{r_{*}}\left|h_{r_{*} r_{*}}\right|^{2}}\right), \\
& \mathcal{I}_{r_{*} d}^{F D}=\mathrm{R}_{\mathrm{EH}-\mathrm{FDJD}}(1-\alpha) \log _{2}\left(1+\frac{P_{s}\left|h_{s d}\right|^{2}+P_{r_{*}}\left|h_{r_{*} d}\right|^{2}}{P_{p}\left|h_{p d}\right|^{2}}\right) .
\end{aligned}
$$

From (11), it can be noted that the signals from $\mathrm{S}$ and $\mathrm{R}_{*}$ are treated as useful information at the destination. Moreover, the self-interference in $\mathrm{R}_{*}$ is taken into account in (10). Thus, the overall outage probability of the proposed scheme EH-FDJD can be written as

$$
O^{\mathrm{EH}-\mathrm{FDJD}}=O_{s d}^{F D}\left(O_{s r_{*}}^{F D}\right)^{U}+\left(1-\left(O_{s r_{*}}^{F D}\right)^{U}\right) O_{r_{*} d}^{F D} .
$$

In order to obtain a closed-form expression for (12), it is necessary to solve the terms individually. The value of $O_{s d}^{F D}$ can be obtained using (7), with $\mathrm{R}_{\mathrm{EH}-\mathrm{FDJD}}=1$ and the outage probability $O_{s r_{*}}^{F D}=\operatorname{Pr}\left[\mathcal{I}_{s r_{*}}<\mathcal{R}\right]$ can be obtained as:

$$
O_{s r_{*}}^{F D}=\operatorname{Pr}\left[\log _{2}\left(1+\frac{\mathcal{K}\left|h_{p s}\right|^{2}\left|h_{s r_{*}}\right|^{2}}{\left|h_{p r_{*}}\right|^{2}\left(1+\mathcal{K}\left|h_{r_{*} r_{*}}\right|^{2}\right)}\right)<\frac{\mathcal{R}_{\mathrm{EH}-\mathrm{FDJD}}}{(1-\alpha)}\right]
$$

The self-interference term in (13) represented by $\left(\mathcal{K}\left|h_{p r_{*}}\right|^{2}\left|h_{r_{*} r_{*}}\right|^{2}\right)$ can be seen as an attenuated version of the primary interference at the relay. As demonstrated in [14], [19], [31], the self-interference can be attenuated by interference cancellation techniques for residual values in the order of $\delta<10^{-3}$. Thus, we can consider the value of self-interference term $\left(\mathcal{K}\left|h_{r_{*} r_{*}}\right|^{2}<<1\right)$ negligible in (13).
Therefore, the outage probability of the link S-R in (13) can be rewritten as

$$
O_{s r_{*}}^{F D}=\operatorname{Pr}\left[\log _{2}\left(1+\frac{\mathcal{K}\left|h_{p s}\right|^{2}\left|h_{s r_{*}}\right|^{2}}{\left|h_{p r_{*}}\right|^{2}}\right)<\frac{\mathcal{R}_{\text {EH-FDJD }}}{(1-\alpha)}\right] .
$$

Thus, the outage probability $O_{s r_{*}}^{F D}$ can be solved in the same way as $O_{s d}^{D T}$, being expressed as (15) (at the top of next page) where $\mathcal{Z}_{m r}$ is a constant given by:

$$
\mathcal{Z}_{m r}=\frac{\left(2^{\frac{\mathcal{R}_{\text {sch }}}{(1-\alpha)}}-1\right) m_{s r_{*}} \lambda_{p r_{*}}}{\mathcal{K} \lambda_{p s} \lambda_{s r_{*}}} .
$$

The outage probability of the $\mathrm{R}_{*} \rightarrow \mathrm{D}$ link is defined as $O_{r_{*} d}^{F D}=\operatorname{Pr}\left[\mathcal{I}_{r_{*} d}<\mathcal{R}\right]$ and can be approximated as in (17), where $X=\frac{\left(2^{\frac{\mathcal{R}_{\mathrm{sch}}}{(1-\alpha)}}-1\right)}{\mathcal{K}}$ and the terms $\xi_{s}, \xi_{r}, \varrho_{s}$ e $\varrho_{r}$ are given, respectively, by:

$$
\begin{gathered}
\xi_{s}=\left(\frac{\Gamma\left(m_{p s}\right) \Gamma\left(m_{p s}+1\right) \Gamma\left(m_{s d}\right) \Gamma\left(m_{s d}+1\right)}{\Gamma\left(m_{p s}+0.5\right)^{2}+\Gamma\left(m_{s d}+0.5\right)^{2}}-1\right)^{-1}, \\
\xi_{r_{*}}=\left(\frac{\Gamma\left(m_{p r_{*}}\right) \Gamma\left(m_{p r_{*}}+1\right) \Gamma\left(m_{r_{*} d}\right) \Gamma\left(m_{r_{*} d}+1\right)}{\Gamma\left(m_{p r_{*}}+0.5\right)^{2}+\Gamma\left(m_{r_{*} d}+0.5\right)^{2}}-1\right)^{-1}, \\
\varrho_{s}=\sqrt{\frac{\lambda_{p s} \lambda_{s d}}{m_{p s} m_{s d}}} \frac{\Gamma\left(m_{p s}+0.5\right) \Gamma\left(m_{s d}+0.5\right)}{\Gamma\left(m_{p s}\right) \Gamma\left(m_{s d}\right)} \frac{1}{\xi_{s}}, \\
\varrho_{r_{*}}=\sqrt{\frac{\lambda_{p r_{*}} \lambda_{r_{*} d}}{m_{p r_{*}} m_{r_{*} d}}} \frac{\Gamma\left(m_{p r_{*}}+0.5\right) \Gamma\left(m_{r_{*} d}+0.5\right)}{\Gamma\left(m_{p r_{*}}\right) \Gamma\left(m_{r_{*} d}\right)} \frac{1}{\xi_{r_{*}}} .
\end{gathered}
$$

Please, see Appendix A for more details of the derivation.

\section{Energy Harvesting Half-Duplex with Joint-Decoding Scheme}

As a benchmark scheme, we analyze the performance of an Energy Harvesting Half-Duplex Joint Decoding scheme (EHHDJD), whose transmission protocol is described in Fig. 2. The communication occurs in two phases: first, the source broadcast its information to the relays and to destination; and secondly, the selected relay forwards the information to the destination. At the destination both messages are combined and jointly decoded. Consequently, the overall outage probability of the EH-HDJD scheme can be written as:

$$
O^{\mathrm{EH}-\mathrm{HDJD}}=O_{s d}^{H D}\left(O_{s r_{*}}^{H D}\right)^{U}+\left(1-\left(O_{s r_{*}}^{H D}\right)^{U}\right) O_{r_{*} d}^{H D} .
$$




$$
\begin{aligned}
& O_{s r_{*}}^{F D}= \begin{cases}\mathcal{Z}_{m r} e^{\mathcal{Z}_{m r}} \mathrm{E}_{n}\left(m_{s r_{*}}, \mathcal{Z}_{m r}\right), & \text { for } m_{s r_{*}} \geq 1 \text { and } m_{p s}=m_{p r_{*}}=1 \\
1-\frac{\Gamma\left(m_{p s}+m_{\left.p r_{*}\right)}\right)\left(m_{p r_{*}}, 1-m_{p s}, \frac{Z_{m r} m_{p s}}{m_{p r_{*}}}\right)}{\Gamma\left(m_{p s}\right)}, & \text { for } m_{s r_{*}}=1, m_{p s} \geq 1 \text { and } m_{p r_{*}} \geq 1 .\end{cases} \\
& O_{r_{*} d}^{F D}=\operatorname{Pr}\left[\log _{2}\left(1+\mathcal{K}\left(\frac{\left|h_{p s}\right|^{2}\left|h_{s d}\right|^{2}+\left|h_{p r_{*}}\right|^{2}\left|h_{r_{*} d}\right|^{2}}{\left|h_{p d}\right|^{2}}\right)\right)<\frac{\mathcal{R}_{\text {EH-FDJD }}}{(1-\alpha)}\right] \\
& \approx 2^{-\xi_{s}-\xi_{r_{*}}} \varrho_{s}^{-\xi_{s}} \varrho_{r_{*}}^{-\xi_{r_{*}}}\left(X \lambda_{p d}\right)^{\frac{\xi_{s}+\xi_{r_{*}}}{2}} U\left(\frac{\xi_{s}}{2}, 0.5, \frac{X \lambda_{p d}}{4 \varrho_{s}^{2}}\right) U\left(\frac{\xi_{r_{*}}}{2}, 0.5, \frac{X \lambda_{p d}}{4 \varrho_{r_{*}}^{2}}\right)
\end{aligned}
$$

The outage probability $O^{H D}$ can be written as in (7) while the outage probabilities $O_{s r_{*}}^{H}{ }^{S}$ and $O_{r_{*} d}^{H D}$ are obtained as in (15) and (17), respectively, with $\mathrm{R}_{\mathrm{EH}-\mathrm{HDJD}}=\beta=\frac{1}{2}$.

\section{Numerical Results}

In this section, we present some numerical results to investigate the performance of the EH-FDJD scheme compared to the EH-HDJD scheme, both with joint decoding at destination, and also with the EH-DT direct link scheme without cooperation. Monte Carlo simulations were performed to validate the analytical expressions obtained.

We consider $\sigma_{r_{*} r_{*}}^{2}=1$, a path-loss exponent $v=4, \mathcal{R}=1$ bpcu and $\eta=1^{4}$. Furthermore, it is assumed that all relays are in a cluster, i.e., are at the same distance from the other nodes of the network. The primary transmitter $\mathrm{P}$ is located at coordinates $(0,1)$ on the $\mathrm{X}-\mathrm{Y}$ plane. While $\mathrm{S}$, the cluster of relays and $\mathrm{D}$ are located at coordinates $(1,0),(1.5,0)$ and $(2,0)$, respectively. The cluster of relays is located in a straight line between $\mathrm{S}$ and D. Monte Carlo simulations are represented by red circles in the figures that fallows.

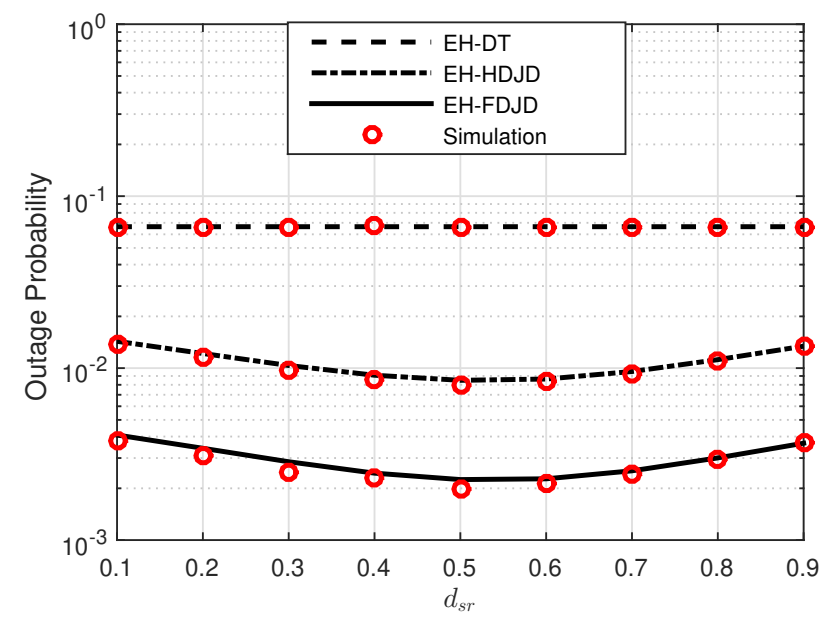

Fig. 4: Outage probability of the different schemes in function of $d_{s r_{*}}$.

First, a scenario with just one cooperative relay and with $m_{i j}=1$ for all channels is analyzed. In order to find the

\footnotetext{
${ }^{4}$ Smaller values for $\eta$ would not change the result of comparisons because the effect would be similar for all schemes and consequently, only the relative position of the results on the $y$ axis would change.
}

optimum position for the relay, the outage probability of the different schemes as a function of the distance between $\mathrm{S}$ and $\mathrm{R}$ is evaluated in Fig.4. We consider $\alpha=0.5$ and that the position of $\mathrm{R}_{*}$ is varied from (1.1.0) to (1.9.0). The direct transmission is just included on the figure for comparison purposes.

From Fig.4, it can be noticed that the Monte Carlo simulations agree very well with the theoretical results. It can also be observed that the EH-FDJD scheme presents the best performance among all the schemes analyzed. Furthermore, it can be seen that the optimum position of the relay is exactly in the midpoint between $S$ and $D$ for this particular scenario. This is because when $R_{*}$ is closer to $S$, the secondary nodes harvest more energy, however the interference from the primary transmitter is large at the relay, causing an increase in the outage probability. When $\mathrm{R}_{*}$ is closer to $\mathrm{D}$, the interference from $\mathrm{P}$ decreases at the relay but at the same time, the energy harvested from the primary transmission also decreases, thereby increasing the outage probability.

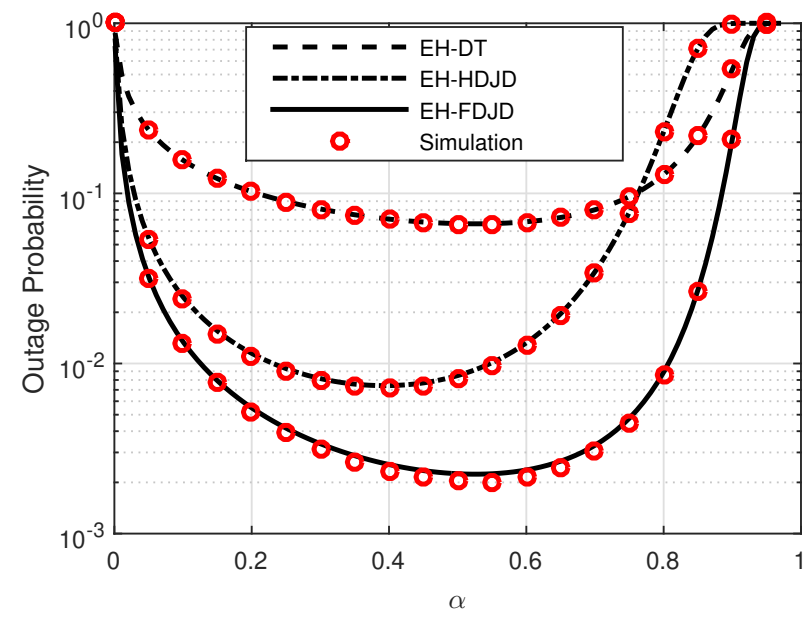

Fig. 5: Outage probability of the different schemes as a function of $\alpha$ with $d_{s r_{*}}=0.5$.

Fig. 5 illustrates the analysis of outage probability as a function $\alpha$. This analysis is important because the value of $\alpha$ influences both power and transmission rate of the secondary nodes. We consider $d_{s r_{*}}=0.5$ which corresponds to the optimal relay position in this scenario as seen in Fig. 4. It can be noted that the proposed scheme has the best performance 
among all schemes for the whole range of $\alpha$. When $\alpha=0.8$ the EH-FDJD scheme achieves an outage probability of $10^{-2}$, while the outage probability of the EH-DT scheme is greater than $10^{-1}$. Moreover, for a given outage probability, the harvesting time may be higher in EH-FDJD than in EHHDJD, so with a larger amount of energy harvested, the proposed scheme is able to obtain a lower outage probability. In addition, the proposed scheme may obtain a certain outage probability with a shorter charging time and thereby it can devote more time to the transmission phase. For instance, with an outage probability of $10^{-2}$, we have $\alpha=0.11$ in EH-FDJD and $\alpha=0.22$ in EH-HDJD.

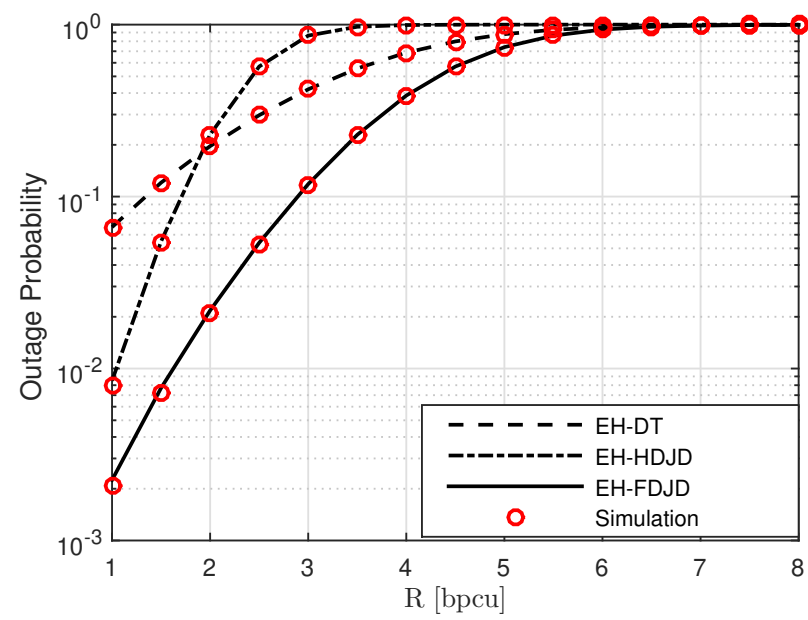

Fig. 6: Outage probability of the different schemes as a function of the expected transmission rate $\mathcal{R}$ with $d_{s r_{*}}=0.5$ and $\alpha=0.5$.

Fig. 6 depicts the outage probability as a function of the expected transmission rate $\mathcal{R}$. In this analysis, we consider $\alpha=0.5$ and $d_{s r_{*}}=0.5$. It can be observed that for a given outage probability, the EH-FDJD scheme achieves even the double of the achievable rate in EH-HDJD. This occurs because in the proposed scheme the relay transmits during all the transmission phase. It can also be observed that the EH-FDJD scheme presents the better performance for all transmission rate values considered in comparison with all other schemes.

In order to investigate the effect of some line-of-sight in the links between the PU transmitter and the secondary source and relay, we next analyze the outage probability as a function of $\alpha$ for different values of the fading parameters $m_{p s}=m_{p r_{*}} \in$ $\{1,2,3\}$ and considering $\mathcal{R}=1 \mathrm{bpcu}$ and $m_{s r_{u}}=m_{s d}=m_{r_{*} d}=$ $m_{p d}=1$. Fig. 7 shows the outage probability of the proposed scheme EH-FDJD in comparison with the EH-HDJD scheme. The EH-DT scheme is not considered, since in previous results it has a much lower performance in comparison with EHHDJD and EH-FDJD.

From Fig. 7, it can be noticed that the system performance improves significantly when compared to the scenario without line-of-sight, a fact fully expected. With some line-of-sight in the links $\mathrm{P} \rightarrow \mathrm{S}$ and $\mathrm{P} \rightarrow \mathrm{R}_{*}$ the channel coefficients $h_{p s}$ and $h_{p r_{*}}$ increase, thus the nodes harvest more energy

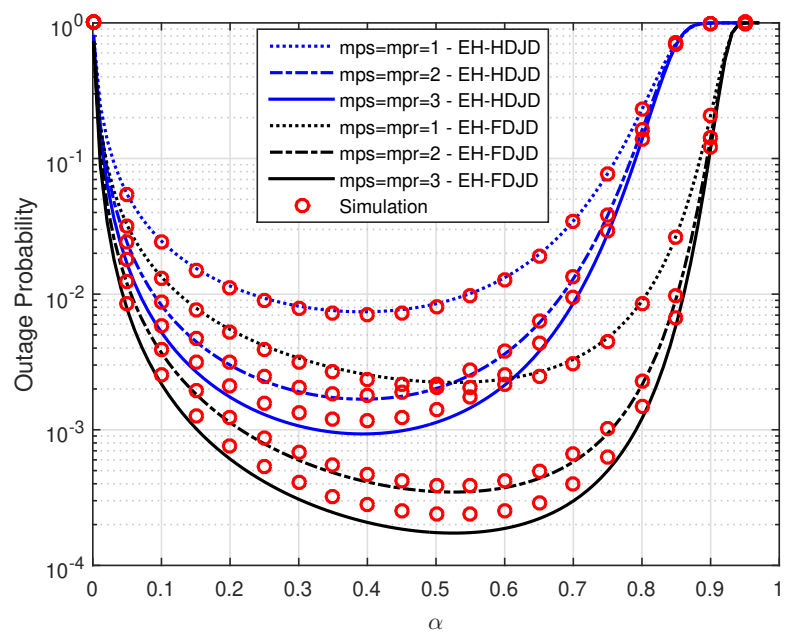

Fig. 7: Outage probability as function of $\alpha$ considering $m_{p s}=$ $m_{p r_{*}}=\{1,2,3\}, m_{s r_{u}}=m_{r_{*} d}=m_{s d}=m_{p d}=1$ and $\mathcal{R}=1$ bpcu

which improves the whole system performance. Even if the interference at the relay has increased, the greater amount of energy harvested improves the performance of the system as a whole. Finally, we can observe that the optimum value of $\alpha$ does not change with variations in the fading parameters $m_{p s}$ and $m_{p r_{*}}$.

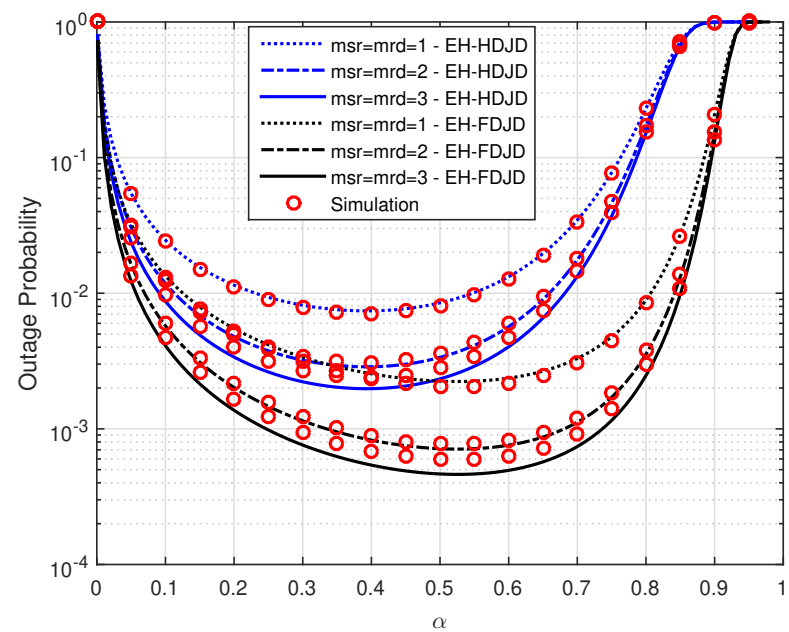

Fig. 8: Outage probability as function of $\alpha$ considering $m_{s r_{u}}=$ $m_{r_{*} d}=\{1,2,3\}, m_{p s}=m_{p r_{*}}=m_{p d}=m_{s d}=1$ and $\mathcal{R}=1$ bpcu

The effect of some line-of-sight in the secondary links $\mathrm{S} \rightarrow$ $\mathrm{R}_{*}$ and $\mathrm{R}_{*} \rightarrow \mathrm{D}$ was next investigated by evaluating the outage probability as a function of $\alpha$ for different fading parameter values $m_{s r_{u}}=m_{r_{*} d} \in\{1,2,3\}$ and considering $\mathcal{R}=1 \mathrm{bpcu}$ and $m_{p s}=m_{p r_{*}}=m_{p d}=m_{s d}=1$.

Fig. 8 shows that the performance of all schemes improves with the increment in the fading parameters. Moreover, when we compare the two scenarios analyzed, it can be noted that 
the secondary network performs better when there is some line-of-sight in the links between the primary and secondary network than when the line-of-sight occurs just in some secondary links. As in the previous analysis, we can observe that the optimum value of $\alpha$ does not change with variations in the fading parameters $m_{s r_{u}}$ and $m_{r_{*} d}$.

In Fig. 9, the outage probability for the proposed scheme is plotted as a function of $\alpha$ varying the number of relays, with $\mathcal{R}=1 \mathrm{bpcu}$ and $m_{i j}=1$ for all links. It is observed that when the number of relays increases, the performance of the systems in terms of outage probability improves. This is because the secondary source has more possibilities to transmit its information, which increases the chances of at least one relay decoding the message and thus guaranteeing a better outage probability.

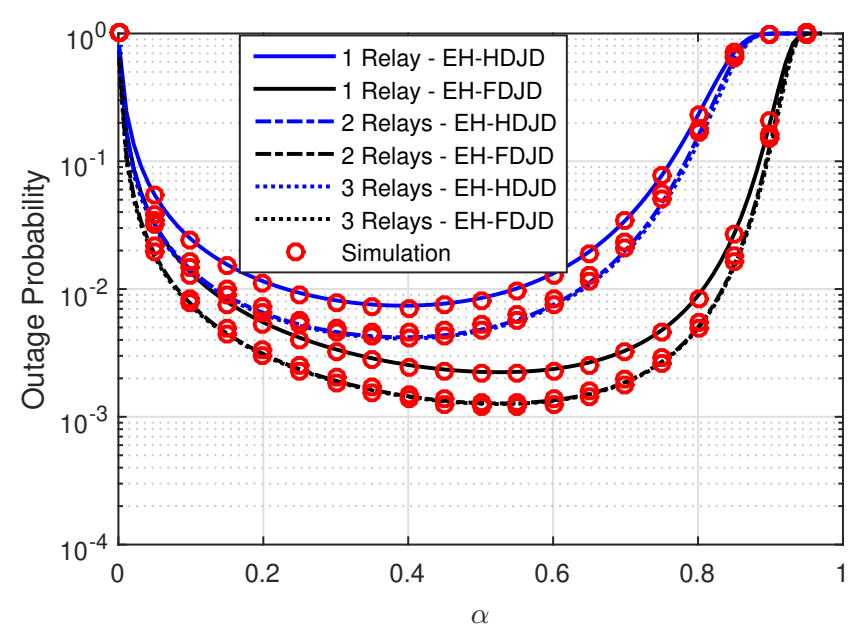

Fig. 9: Outage probability as function of $\alpha$ varying the number of relays, with $m_{i j}=1$ for all channels and $\mathcal{R}=1 \mathrm{bpcu}$.

\section{CONCLUSION}

In this paper, the performance of a full-duplex energy harvesting cooperative cognitive network under Nakagami- $m$ fading was investigated. It was considered that the secondary users harvest energy from the RF signals transmitted by the primary user. Analytical expressions for the outage probability were derived and validated through numerical simulations. The results show that the EH-FDJD scheme has the best performance in terms of outage probability, when compared with EH-HDJD scheme and EH-DT direct transmission for the proposed particular scenario. Moreover, it was shown that the performance of the system improves when there is more than one cooperating relay.

\section{APPENDIX A \\ DERIVATION OF $O_{r_{*} d}^{F D}$}

In order to find the outage probability for the link $\mathrm{R}_{*} \rightarrow \mathrm{D}$ in (17), we will use the results presented in [31] to approximate the product of two random variables Nakagami$m\left(Z_{k}=h_{p k} h_{k d}\right)$ by one Gamma variable $\left(\Lambda_{k} \sim \Gamma\left(\xi_{k}, \varrho_{k}\right)\right)$, where $k \in\left\{s, r_{*}\right\}$, whose parameters $\xi_{k}$ e $\varrho_{k}$ can be estimated through the moment generator method described in [32]. To this end, we define the first and second moments of $\Lambda$ as $E\left[\Lambda_{k}\right]=\xi_{k} \varrho_{k}$ and $E\left[\Lambda_{k}^{2}\right]=\xi_{k}\left(1+\xi_{k}\right) \varrho_{k}^{2}$ [32]. From [33, Eq. 9], the $n$ th-order moment of $Z_{k}$ can be determined as

$$
E\left[Z_{k}^{n}\right]=\frac{\Gamma\left(m_{p k}+\frac{n}{2}\right) \Gamma\left(m_{k d}+\frac{n}{2}\right)}{\Gamma\left(m_{p k}\right) \Gamma\left(m_{k d}\right)}\left(\frac{\lambda_{p k}}{m_{p k}}\right)^{\frac{n}{2}}\left(\frac{\lambda_{k d}}{m_{k d}}\right)^{\frac{n}{2}} .
$$

Then, by matching $E\left[Z_{k}\right]$ with $E\left[\Lambda_{k}\right]$ and $E\left[Z_{k}^{2}\right]$ with $E\left[\Lambda_{k}^{2}\right]$ and after some algebraic manipulations, $\xi_{k}$ and $\varrho_{k}$ can be written as a function of the parameters of the random variable Nakagami- $m \Gamma_{s}\left(\xi_{s}, \varrho_{s}\right)$ and $\Gamma_{r_{*}}\left(\xi_{r_{*}}, \varrho_{r_{*}}\right)$.

Consequently, considering $Z_{k}^{2} \approx\left|h_{p k}\right|^{2}\left|h_{k d}\right|^{2}$ and solving the integral in (24), the outage probability $O_{r_{*} d}^{F D}$ can be written as in (17).

\section{REFERENCES}

[1] H. D. Pereira, S. B. Mafra, and E. M. G. Fernández, "Análise de desempenho de uma rede cognitiva full-duplex com captação de energia," XXXV Brazilian Telecommuncation Symposium (SBrT'17) - São-Pedro SP, Brasil, pp. 1-5, September 2017.

[2] J. Mitola and G. Q. Maguire, "Cognitive radio: making software radios more personal," IEEE Personal Communications, vol. 6, no. 4, pp. 1318, August 1999, doi: 10.1109/JPROC.2010.2070470.

[3] A. Goldsmith, S. A. Jafar, I. Maric, and S. Srinivasa, "Breaking spectrum gridlock with cognitive radios: An information theoretic perspective," Proceedings of the IEEE, vol. 97, no. 5, pp. 894-914, May 2009, doi: 10.1109/JPROC.2009.2015717.

[4] S. Sudevalayam and P. Kulkarni, "Energy harvesting sensor nodes: Survey and implications," IEEE Communications Surveys Tutorials, vol. 13, no. 3, pp. 443-461, March 2011, doi: 10.1109/SURV.2011.060710.00094.

[5] R. Amirtharajah and A. P. Chandrakasan, "Self-powered signal processing using vibration-based power generation," IEEE Journal of Solid-State Circuits, vol. 33, no. 5, pp. 687-695, May 1998, doi: 10.1109/4.668982

[6] S. Chalasani and J. M. Conrad, "A survey of energy harvesting sources for embedded systems," in IEEE SoutheastCon 2008, April 2008, pp. 442-447, doi: 10.1109/SECON.2008.4494336.

[7] L. R. Varshney, "Transporting information and energy simultaneously," in 2008 IEEE International Symposium on Information Theory, July 2008, pp. 1612-1616, doi: 10.1109/ISIT.2008.4595260.

[8] X. Zhou, R. Zhang, and C. K. Ho, "Wireless information and power transfer: Architecture design and rate-energy tradeoff," in 2012 IEEE Global Communications Conference (GLOBECOM), December 2012, pp. 3982-3987, doi: 10.1109/GLOCOM.2012.6503739.

[9] R. Zhang and C. K. Ho, "Mimo broadcasting for simultaneous wireless information and power transfer," IEEE Transactions on Wireless Communications, vol. 12, no. 5, pp. 1989-2001, May 2013, doi: 10.1109/TWC.2013.031813.120224.

[10] A. A. Nasir, X. Zhou, S. Durrani, and R. A. Kennedy, "Relaying protocols for wireless energy harvesting and information processing," IEEE Transactions on Wireless Communications, vol. 12, no. 7, pp. 3622-3636, July 2013, doi: 10.1109/TWC.2013.062413.122042.

[11] J. N. Laneman, D. N. C. Tse, and G. W. Wornell, "Cooperative diversity in wireless networks: Efficient protocols and outage behavior," IEEE Transactions on Information Theory, vol. 50, no. 12, pp. 3062-3080, December 2004, doi: 10.1109/TIT.2004.838089.

[12] A. Nosratinia, T. E. Hunter, and A. Hedayat, "Cooperative communication in wireless networks," IEEE Communications Magazine, vol. 42, no. 10, pp. 74-80, October 2004, doi: 10.1109/MCOM.2004.1341264.

[13] G. Kramer, M. Gastpar, and P. Gupta, "Cooperative strategies and capacity theorems for relay networks," IEEE Transactions on Information Theory, vol. 51, no. 9, pp. 3037-3063, September 2005, doi: 10.1109/TIT.2005.853304.

[14] M. Duarte, C. Dick, and A. Sabharwal, "Experiment-driven characterization of full-duplex wireless systems," IEEE Transactions on Wireless Communications, vol. 11, no. 12, pp. 4296-4307, December 2012, doi: 10.1109/TWC.2012.102612.111278. 


$$
\begin{aligned}
& O_{r_{*} d}^{F D}=\operatorname{Pr}\left[\log _{2}\left(1+\mathcal{K}\left(\frac{\left|h_{p s}\right|^{2}\left|h_{s d}\right|^{2}+\left|h_{p r_{*}}\right|^{2}\left|h_{r_{*}}\right|^{2}}{\left|h_{p d}\right|^{2}}\right)\right)<\frac{\mathcal{R}_{\mathrm{EH}-\mathrm{FDJD}}}{(1-\alpha)}\right]
\end{aligned}
$$

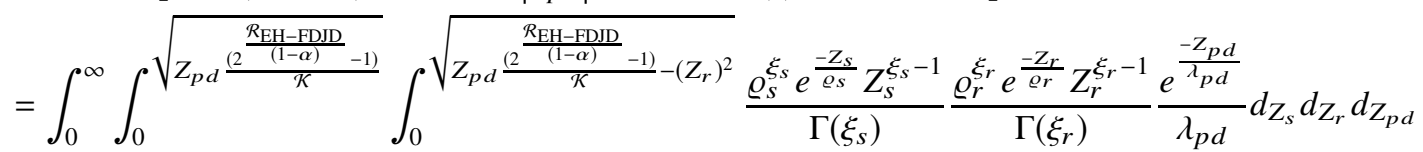

[15] T. Riihonen, S. Werner, and R. Wichman, "Mitigation of loopback self-interference in full-duplex mimo relays," IEEE Transactions on Signal Processing, vol. 59, no. 12, pp. 5983-5993, December 2011, doi: 10.1109/TSP.2011.2164910.

[16] S. Atapattu, H. Jiang, J. Evans, and C. Tellambura, "Time-switching energy harvesting in relay networks," in 2015 IEEE International Conference on Communications (ICC), June 2015, pp. 5416-5421, doi: 10.1109/ICC.2015.7249185.

[17] C. Zhong, H. A. Suraweera, G. Zheng, I. Krikidis, and Z. Zhang, "Wireless information and power transfer with full duplex relaying," IEEE Transactions on Communications, vol. 62, no. 10, pp. 3447-3461, October 2014, doi: 10.1109/TCOMM.2014.2357423.

[18] T. Q. Duong, P. L. Yeoh, V. N. Q. Bao, M. Elkashlan, and N. Yang, "Cognitive relay networks with multiple primary transceivers under spectrum-sharing," IEEE Signal Processing Letters, vol. 19, no. 11, pp. 741-744, November 2012, doi: 10.1109/LSP.2012.2217327.

[19] S. B. Mafra, H. Alves, D. B. Costa, R. D. Souza, E. M. G. Fernandez, and M. Latva-aho, "On the performance of cognitive full-duplex relaying under spectrum sharing constraints," EURASIP Journal on Wireless Communications and Networking, vol. 2015, no. 1, pp. 1-13, 2015, doi: 10.1186/s13638-015-0397-z. [Online]. Available: http://dx.doi.org/10.1186/s13638-015-0397-z

[20] Y. Liu, S. A. Mousavifar, Y. Deng, C. Leung, and M. Elkashlan, "Wireless energy harvesting in a cognitive relay network," IEEE Transactions on Wireless Communications, vol. 15, no. 4, pp. 2498-2508, April 2016, doi: 10.1109/TWC.2015.2504520.

[21] S. Luo, R. Zhang, and T. J. Lim, "Optimal save-then-transmit protocol for energy harvesting wireless transmitters," IEEE Transactions on Wireless Communications, vol. 12, no. 3, pp. 1196-1207, March 2013, doi: 10.1109/TWC.2013.012413.120488.

[22] S. B. Mafra, E. M. G. Fernández, S. Montejo-Sánchez, and H. D. Pereira, "Performance analysis of energy constrained cognitive fullduplex generalized network coding scheme," XXXV Simpósio Brasileito de Telecomunicações e Processamento de Sinais (SBrT), September 2017.

[23] M. Abramowitz, Handbook of Mathematical Functions, With Formulas, Graphs, and Mathematical Tables,. Dover Publications, Incorporated, 1974, isbn: 0486612724.

[24] I. S. Gradshteyn and I. M. Ryzhik, Table of integrals, series, and products, 7th ed. Elsevier/Academic Press, Amsterdam, 2007.

[25] V. Blagojevic and P. Ivanis, "Ergodic capacity for tas/mrc spectrum sharing cognitive radio," IEEE Communications Letters, vol. 16, no. 3, pp. 321-323, March 2012, doi: 10.1109/LCOMM.2012.011312.111488.

[26] F. A. Khan, K. Tourki, M. S. Alouini, and K. A. Qaraqe, "Outage and ser performance of spectrum sharing system with tas/mrc," in 2013 IEEE International Conference on Communications Workshops (ICC), June 2013, pp. 381-385, doi: 10.1109/ICCW.2013.6649263.

[27] J. Men, J. Ge, and C. Zhang, "A joint relay-and-antenna selection scheme in energy-harvesting mimo relay networks," IEEE Signal Processing Letters, vol. 23, no. 4, pp. 532-536, April 2016, doi: 10.1109/LSP.2016.2538290.

[28] A. Bletsas, A. Lippnian, and D. P. Reed, "A simple distributed method for relay selection in cooperative diversity wireless networks, based on reciprocity and channel measurements," in 2005 IEEE 61st Vehicular Technology Conference, vol. 3, May 2005, pp. 1484-1488 Vol. 3, doi: 10.1109/VETECS.2005.1543566.

[29] A. Bletsas, H. Shin, and M. Z. Win, "Cooperative communications with outage-optimal opportunistic relaying," Trans. Wireless. Comm., vol. 6, no. 9, pp. 3450-3460, September 2007, doi: 10.1109/TWC.2007.06020050. [Online]. Available: http://dx.doi.org/10.1109/TWC.2007.06020050

[30] M. Khafagy, A. Ismail, M. S. Alouini, and S. Aissa, "On the outage performance of full-duplex selective decode-and-forward relaying," IEEE
Communications Letters, vol. 17, no. 6, pp. 1180-1183, June 2013, doi: 10.1109/LCOMM.2013.042313.130444.

[31] H. Alves, D. B. da Costa, R. D. Souza, and M. Latva-aho, "Performance of block-markov full duplex relaying with self interference in nakagamim fading," IEEE Wireless Communications Letters, vol. 2, no. 3, pp. 311-314, June 2013, doi: 10.1109/WCL.2013.030613.130023.

[32] A. Papoulis, Probability, Random Variables, and Stochastic Processes, ser. McGraw-Hill Series in Electrical Engineering. McGraw-Hill, 1984, isbn: 9780070484689

[33] G. K. Karagiannidis, N. C. Sagias, and P. T. Mathiopoulos, "N*nakagami: A novel stochastic model for cascaded fading channels," IEEE Transactions on Communications, vol. 55, no. 8, pp. 1453-1458, August 2007, doi: 10.1109/TCOMM.2007.902497.

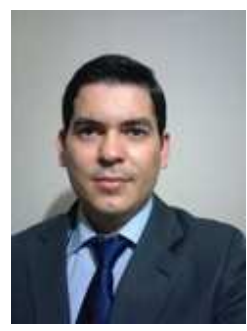

Hebert Douglas Pereira was born in Ouvidor-GO, Brazil, in 1982. He received the B.Sc. degree in telecommunication engineering from the Faculdade Pitágoras de Uberlândia, Uberlândia-MG, Brazil, in 2006, the M.Sc. degree from the Federal University of Paraná (UFPR), Curitiba, Brazil, in 2017. His research interests lie in the broad area of wireless communication theory, with applications to cognitive radio networks, energy harvesting and cooperative communications. $\mathrm{He}$ is a student member of the Brazilian Telecommunications Society (SBrT).

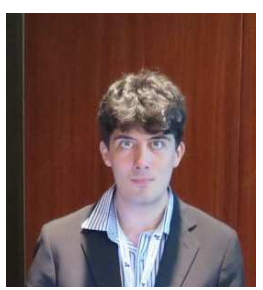

Samuel Baraldi Mafra was born in CuritibaPR, Brazil, in 1986. He received the B.Sc. degree from the Santa Catarina State University (UDESC), Joinville, Brazil, in 2010, the M.Sc. degree from the Federal University of Paraná (UFPR), Curitiba, Brazil, in 2012 and the D.Sc. degree from the Federal University of Technology - Paraná (UTFPR), Curitiba, Brazil, in 2015, all in electrical engineering. Since September 2015, he has held a PostDoctoral position at the UFPR. His research interests lie in the broad area of wireless communication theory, with applications to cognitive radio networks and cooperative communications. He is a student member of the IEEE Communications Society and Brazilian Telecommunications Society (SBrT).

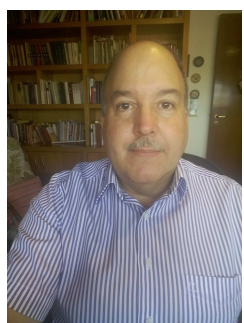

Evelio M. G. Fernandez (M'13) was born in Santa Clara, Cuba, in 1962. He received the B.Sc. degree in Electrical Engineering from the Central University of Las Villas (UCLV), Cuba, in 1985 and his M.Sc. and D.Sc. degrees in Electrical Engineering from the State University of Campinas (UNICAMP), Brazil in 1997 and 2001, respectively. He is currently an Associate Professor at the Department of Electrical Engineering at the Federal University of Paraná (UFPR), Brazil. His research interests include channel coding techniques, digital communications, wireless networks and cognitive radio systems. He is a Member of the IEEE and of the Brazilian Telecommunications Society (SBrT). He is a co-recipient of the 2016 Research Award from the Cuban Academy of Sciences. 